\title{
The DELPHI Trigger System at LEP2 Energies
}

The DELPHI Trigger Group

A. Augustinus ${ }^{1}$, V. Canale ${ }^{2}$, P. Charpentier ${ }^{1}$, J-P. De Wulf ${ }^{3}$,

F. Fontanelli ${ }^{4}$, F. Formenti ${ }^{1}$, C. Gaspar ${ }^{1}$, P. Gavillet ${ }^{1}, R$. Goorens ${ }^{3}$, J.P. Laugier ${ }^{5}$, P. Musico ${ }^{4}$,M. Paganoni ${ }^{6}$, M. Sannino ${ }^{4}$ and G. Valenti ${ }^{7}$ 1 CERN, Geneva, Switzerland

2 Dipartimento di Fisica Universitá di Roma Tor Vergata and INFN, Rome, Italy

3 I.I.H.E., Brussels,Belgium

4 Dipartimento di Fisica Università di Genova and INFN, Genova, Italy

5 DAPNIA, Saclay, France

6 Dipartimento di Fisica Universitá di Milano and INFN, Milano, Italy

7 Sezione INFN di Bologna, Bologna, Italy

\begin{abstract}
In this paper we describe the modifications carried out on the DELPHI trigger complex since the beginning of the high energy runs of LEP. The descriptions of the trigger configurations and performances for the 2000 data taking period are also presented.
\end{abstract}




\section{Introduction and Physics motivations}

Since the increase of the LEP beam energy in 1995, a number of upgrades and modifications of the DELPHI trigger complex have been accomplished. While some hardware interventions were dedicated to replacing and rebuilding Fastbus modules with improved capabilities, the major change occurred in the trigger conditions, mostly dictated by the physics to be addressed at energies above the Z. A further improvement was the final implementation of all four levels in the trigger, which substantially reduced the amount of data that passed through the analysis chain.

As a reminder, figure 1 shows schematically the geometrical ${ }^{1}$ arrangement of the subdetectors relevant for the trigger system; their detailed description may be found in [1]. There are tracking chambers (ID, OD, TPC, FCA, FCB, MUB and MUF), calorimeters (HPC, FEMC, HAB, HAF and STIC) and scintillator counters (TOF and HOF). The trigger system is structured into four successive levels. T1 and T2, being hardware triggers, are synchronous with respect to the Beam Cross Over (BCO), while T3 and T4 are software filters performed asynchronously with respect to BCO. At high energy the time between successive collisions is $\approx 22 \mu \mathrm{s}$, the $\mathrm{T} 1$ decision is formed within $5 \mu \mathrm{s}$ of the $\mathrm{BCO}$ and only applies loose requirements. The T2 decision is formed within $39 \mu \mathrm{s}$ of the BCO. The trigger information at this level is more refined and the data from detectors with long drift time (TPC and HPC) can also be considered. The third level, T3, has been designed as a software trigger with the important goal of enriching the physics yield of the data written on tape. Although the original aim of T4 was to tag rare physics candidates, its task at high LEP energies was to filter out events passed by T1, T2 and T3 with reduced physics information. The actual online tagging was performed asynchronously from the main data stream.

A good trigger system should have high efficiency to expected standard physics pro-

$$
{ }_{26}^{25} \text { Whic }
$$



the beginning of the LEP high energy runs in 1996 the trigger configuration was very similar to that of the previous years [4].

\begin{tabular}{|l|c|c|}
\hline Z-calibration runs & $\mathrm{T} 1(\mathrm{~Hz})$ & $\mathrm{T} 2(\mathrm{~Hz})$ \\
\hline 1996 data taking & $\sim 500$ & $\sim 5.0$ \\
2000 data taking & $\sim 250$ & $\sim 3.5$ \\
\hline
\end{tabular}

Table 1: Comparison of trigger rates for Z-running in the years 1996 and 2000. They correspond to fills with similar machine conditions.

50

51

therefore geared towards a reduction in the trigger rate, with minimal efficiency loss for standard physics channels, and a retention of a reasonable sensitivity to potential new physics. To illustrate the effect on the rate due to the change in the trigger conditions we report in table 1 the first and second level rates for typical fills during the Z-calibration runs in the years 1996 and 2000. The machine conditions across these calibration runs were very similar; in particular the total beam current was $\sim 3.5 \mathrm{~mA}$ in both cases. At

In the following we review the hardware and performance of the DELPHI trigger. In section 2 we describe the modifications to the architecture, while section 3 is dedicated to the description of the trigger conditions. In section 4 we discuss the physics performance of the system. Our conclusions are formed in section 5 .

\section{Architecture update}

A detailed hardware description of the DELPHI Central Trigger System may be found in [1]. Two main functionalities are identified: one is the the synchronization of the various components of the system wand the other is the trigger decision procedure. In DELPHI the correct timing is dictated for the all experiment by one module called ZEUS, and locally, for each subdetector, by another module called PANDORA. The logic of the trigger decision is managed by another module called PYTHIA [5]. A schematic representation of the system is shown in figure 2. Since 1996 several modifications were introduced in both sectors in order to improve the capabilities of the system.

\subsection{The Timing Control System}

ZEUS has the main task of controlling the timing of the experiment. It produces the warning signal WNG_BCO about $6 \mu$ s before the BCO signal only when all the data from the previous event have been saved. Since 1998 an upgraded module, NEW ZEUS, has been operational in the experiment. It is a FASTBUS module with all the functions of the old system and some new features. The most important one is the possibility to run without the presence of beams circulating in the machine from which normally the proper signal for synchronization are obtained. The NEW ZEUS is implemented in the more modern technology of the Field Programmable Gate Arrays (FPGA) of the Xilinx Series 3100 type. The timing signals, distributed to the other modules are produced as output functions of a synchronous state machine operated at $20 \mathrm{MHz}$. Appropriate sequences of states are produced, in accordance with the trigger decisions generated by the PYTHIA modules. A detailed description of the sequence may be found in [1].

All the signals from ZEUS are converted to ECL differential levels before being distributed to the subsystems. This process is accomplished in two steps: first the signals 
from ZEUS are converted to ECL and split on 4 lines, then each line is split again 10 times . A dedicated Fan-out board has been developed to operate with the NEW ZEUS.

All the necessary high frequency circuits, i.e. the receiver of the LEP RF, the LEP pickup and the internal oscillator to run in internal synchronization, are implemented in a dedicated module called the Zeus Daughter Board (ZEUS DB). Since 1999 a new version of it has been operational, it is implemented with digital components only and, as a result, it was much more reliable. The card generates, from the circulating beam pickup signal, the CLK_BCO and WNG_BCO signals which are timed with the next BCO. The CLK_BCO is always present while the WNG_BCO is generated only when the system is ready for acquisition. These two signals are obtained by delaying the beam pickup signal by an appropriate number of LEP RF periods. A special division stage has been realized, to produce the RF3 signal (the LEP RF divided by 3 used by subdetectors for local synchronization) in phase with the pickup itself. The RF3 output signal is distributed by optical fibers to the different subdetectors where it is synchronized with the CLK_BCO. The new design allows the monitoring from the front panel of the most important signals, namely: the LEP RF, the beam pickup, the RF3, the CLK_BCO and the WNG_BCO. The system can also run in a mode where the pickup or both the RF and the pickup are produced internally. The period of the local oscillator is close to the LEP RF period. When running without synchronization from the beams in LEP, the CLK_BCO is directly generated from the RF.

The synchronization of DELPHI to the BCO is obtained by detecting the circulating electron and positron bunches with pickup probes installed in front of the focusing quadrupole at a distance of about 40 meters from the detector endcaps. The arrival time of this signal is used, after a proper delay that is loaded in the trigger supervisor ZEUS, to synchronize DELPHI with the BCO. This set-up was used until 1998. Despite the careful set-up, a significant jitter was occasionally observed. This was due to variations in the shape and amplitude of the analog pickup signals form corresponding variations in the intensity and position of the beams. To overcome this problem the LEP injector signal was used. This signal is carefully timed with the beam pickup signal and fed in place of it at the input of the ZEUS DB. This new synchronization system has played an important role in the improvement of the DELPHI data taking efficiency since 1999.

\subsection{The Decision Control System}

The trigger decision in DELPHI is made, both at the first and the second level, in the PYTHIA modules which collect together all the subtrigger results. In general, the local trigger information is expressed as a multiplicity of elements (track segments, energy clusters, etc...) specific to each subdetector. This multiplicity is coded on two bits $(0,1,2,>$ 2) and is sent to the PYTHIA modules through the Trigger Data Lines (TDLs). Each PYTHIA module is composed of 3 stages of programmable Look-up Tables (LUTs). The logical combination between the 120 TDL inputs, corresponding to the trigger conditions, can be programmed and is loaded into the module at the beginning of data taking. The final PYTHIA stage consists of 16 independent outputs (called functions) which can be masked or scaled ${ }^{2}$ individually before being combined in the final trigger decision. This set-up provides very high flexibility and adaptability to several different conditions (LEP background, subdetector malfunction, etc...). The PYTHIA functions of the first level trigger are also transferred to the input of the second level in order to trigger at T2 with individual T1 results.

${ }^{2}$ The maximum scaling factor is 256 . 
A dedicated module, the DECODER BOX, has been realized to receive the TDLs from all subdetectors and to duplicate them. One copy is sent to the PYTHIAs and the other set to a scaler system to monitor the TDL rates. One FASTBUS crate is reserved for a set of 13 scaler modules for online monitoring. Each scaler contains 32 independent channels that provide 32 bits of scaling. It is the possible to monitor through the scalers all the PYTHIA inputs and other special signals. Among these signals are: the luminosity monitors, several machine background monitors (currents in tracking systems, radiation in the silicon microvertex detector, scintillator counters, etc.) and the LEP status signals (luminosity tuning, miniramp, etc...). The background monitors are used to ensure good quality of the collected data, and also to possibly trigger a beam dump system implemented to protect the microvertex detector from radiation damage.

\subsection{The Central Software}

Some modifications were made to the central software of the trigger system. A trigger display is available in the framework of the DELPHI User Interface package (DUI). This display shows the main trigger quantities like the rate of each PYTHIA function. It also provides control functions like loading of the PYTHIA tables, setting scaling factors, enabling/disabling functions, etc. Other quantities displayed are the experiment livetime, the subdetector readout times, the rates of all the components of the scaler system and the overall trigger system status.

\section{$3 \quad$ Trigger Conditions}

As discussed in section 1, the trigger conditions are set with two goals in mind: to maximize the efficiency for standard physics, and to keep enough sensitivity to potentially new physics. The acquisition of an event is decided on the basis of the two successive synchronous levels. The third level trigger, asynchronous with the BCO, validates events before they are forwarded to storage. This level uses the same logic as the level-2 trigger complemented with more detailed quantities derived from the detector data themselves. Since 1997 a fourth level trigger, based on the production version of the offline reconstruction program, has also been introduced to eliminate events with reduced physics information. Only the first and second levels are relevant for the online live-time of the experiment (rates, dead-time, etc.). The third and fourth levels oly reduce the number of events to be processed offline.

\subsection{Configuration During the High Energy Runs}

Two sets of trigger conditions were used during the high energy runs, one for physics events and one for calibration/test.

At the first level, the trigger for physics events is any one of the following conditions:

- at least one track segment in one or more of the tracking devices ID, OD, TPC, FCA and FCB;

- at least one energy cluster above threshold in one of the calorimeters HPC, FEMC, $\mathrm{HAB}$ and HAF;

- at least one track segment in the muon chambers (MUB);

- a back-to-back coincidence of scintillators (TOF and HOF). 
In general, detectors have separate first and second level processors ${ }^{3}$. At the second level the trigger conditions generally involve coincidences between different subdetectors.

The components are summarized in table 2. Standard conditions normally include:

- at least one track segment in the barrel region. This is the coincidence between ID, $\mathrm{OD}$ and MUB/HAB and the TPC contiguity trigger, CTG [6]. This trigger uses pad information and requires single tracks with a nominal transverse momentum threshold which can be varied between $0.6-1.6 \mathrm{GeV} / \mathrm{c}$. To cover the cracks between different TPC sectors a dedicated component has been implemented, the $\mathrm{IDOD}_{6}$, which is a coincidence of ID and OD detectors in these geometrical regions;

- at least one track segment in the forward (backward) angular regions. In these regions several subtriggers are available for track segments or energy deposit: 1) TRFW(BW) which is a coincidence of FCA and FCB in the forward (backward) region, 2) TPCFW(BW) is a dedicated trigger processor which uses information from the TPC wires and it acts in the forward (backward) region, 3) EMFW(BW) is the electromagnetic trigger in the forward (backward) regions obtained with the FEMC calorimeter, 4) MUFW and MUBW are the triggers from muon chambers in the forward and backward regions, 5) HAFW and HABW are the triggers from the forward and backward hadron calorimeters. Only coincidences of these sub-triggers are considered to reduce the rate;

- at least one electromagnetic shower above threshold in the barrel, forward or backward regions. In the barrel HPC subtrigger both scintillator and chamber data are used;

- at least one neutral shower in the STIC. Charged showers are vetoed by scintillator counters in front of the luminometer;

- at least two electromagnetic clusters in the barrel region. This trigger, conceived for multi-photon final states, is obtained from the HPC scintillator data only. The energy threshold and geometrical acceptance are somewhat different from the previous single photon barrel trigger;

- a back-to-back coincidence of the barrel scintillators TOFBB. This component increases the redundancy in particular for dilepton and multiphoton events and is useful to estimate efficiency. Due to rate problems it was occasionally prescaled.

Several calibration and test triggers are provided for detector monitoring. These components are usually active at the first level and then transported to the second level to trigger the readout. They are:

- the STIC BHABHA, the trigger for Bhabha events, which is the coincidence of energy deposits in the two arms of the STIC calorimeter in back-to-back topology. This the base trigger for the luminosity measurement;

- the VSAT BHABHA, analogous to the previous but with the VSAT calorimeter, is the trigger for Bhabhas events at very small polar angles;

- the STIC single arm trigger, used to evaluate the efficiency of the BHABHA trigger and to cross-check the small angle neutral trigger;

- the parallel muon trigger obtained by the coincidence of the forward and backward muon chamber quadrants which corresponds to horizontal tracks:

- the TPC laser trigger for calibrations;

- the random trigger, obtained with a ${ }^{55} \mathrm{Fe}$ radioactive source, very useful to run without beam in LEP. A small fraction of random triggers are also kept during

${ }^{3}$ In several cases this is not true and the first level signals are used at the second level (e.g. the hadron calorimeter, the barrel muon chambers and the forward-backward chambers). 


\begin{tabular}{|c|c|c|c|}
\hline Trigger & Barrel Region & Forward Region & Backward Region \\
\hline \multirow[t]{8}{*}{ Single Track } & TPC CTG & ID*HAFW & ID*HABW \\
\hline & $\mathrm{IDOD}_{6}(\mathrm{TPC}$ cracks $)$ & ID*MUFW & ID*MUBW \\
\hline & $\mathrm{ID}^{*} \mathrm{OD}{ }^{*} \mathrm{HABL}$ & TPCFW*EMFW(low) & TPCBW*EMBW(low) \\
\hline & ID*OD*MUBL & TPCFW*MUFW & TPCBW*MUBW \\
\hline & & TPCFW*HAFW & TPCBW*HABW \\
\hline & & TRFW*EMFW(low) & TRBW*EMBW(low) \\
\hline & & TRFW*MUFW & TRBW*MUBW \\
\hline & & TRFW*HAFW & TRBW*HABW \\
\hline \multirow[t]{2}{*}{ Single Photon } & HPC(scint.+chambers) & EMFW (high) & EMBW(high) \\
\hline & & STIC neutral & STIC neutral \\
\hline Multi Photons & HPC $\geq 2$ (scintillator) & - & - \\
\hline
\end{tabular}

Table 2: Trigger components at second level. With respect to the subdetector names defined in figure 1 the following abbreviations are defined for the trigger: 1) TRFW(BW) is a coincidence of FCA and FCB in the forward (backward) region, 2) TPCFW(BW) is a dedicated trigger processor which uses information from the TPC wires and acts in the forward (backward) region, 3) TPC CTG is the TPC contiguity trigger which uses the pad information, 4) EMFW(BW) is the electromagnetic trigger in the forward (backward) regions obtained with the FEMC calorimeter, 5) MUFW, MUBW, MUBL are the triggers from muon chambers in the forward, backward and barrel regions respectively, 6) HAFW, HABW, HABL are the triggers from hadron calorimeters in the forward, backward and barrel regions respectively.

physics data taking.

Scaling factors allow the rates of these components to be controlled individually. One very

\subsection{Trigger Rates, Dead-Time and Stability}

Much effort has been devoted to reduce the experiment dead-time. The fraction of dead-timeintroduced by the trigger can be expressed as function of first and second level trigger rates by the formula:

$$
\alpha_{\text {Trigger }}=\nu_{L E P}^{-1}\left(N_{\text {lost }}^{T 1} \times T 1+N_{\text {lost }}^{T 2} \times T 2\right)
$$

where $\nu_{L E P}$ is the LEP crossing rate and $T 1, T 2$ are first and second level trigger rates (in Hz). $N_{\text {lost }}^{T 1, T 2}$ are the number of BCOs lost due to a T1,T2_YES occurrence. For the standard running at high energy of LEP in 4-bunch mode and an average read-out time 
of $\sim 2.5 \mathrm{~ms}$, we obtain:

$$
\alpha_{\text {Trigger }}(\%) \approx 0.0022 \times T 1+0.22 \times T 2 .
$$

The trigger rate depends on backgrounds which in turn depend on the current in the machine. Figure 4 shows the trigger rates at first and second level as a function of the total current in LEP. The plots correspond to typical fills during the year 2000 $(\sqrt{s} \approx 206 \mathrm{GeV})$. The dead-time induced by the trigger, as a function of the current, is also shown in the figure 4 . In standard conditions the dead-time introduced by the trigger is small $\alpha_{\text {Trigger }} \approx 2 \%$.

The stability of the system over time is monitored with plots of the relevants variables, e.g. the trigger rates, dead-time, read-out, as a function of time on a fill-by-fill basis. A systematic offline analysis of trigger efficiency is performed continuously on limited amounts of integrated luminosity. This procedure is automated and implemented as part of the central offline data quality checking [4]. As an example, figure 5 reports for the 1999 data taking period, the stability of the efficiency ${ }^{4}$ of single track triggers as a function of the integrated luminosity. The bin width is $\sim 1 \mathrm{pb}^{-1}$ in order to have sufficient statistics and corresponds to $\sim 1$ day of data taking with stable machine conditions. The low points correspond to occasional malfunctionings of some subdetectors.

\subsection{The T3 Trigger}

In order to reduce further the rate of events recorded, we have implemented another level in the trigger selection, T3, the level-3 software trigger. T3 uses the same trigger conditions of T2 and therefore preserves the physical features of the events triggered by T2. Each sub-detector participating in T2 performs also a fast analysis (LT3P) of its raw data. The result of this processing is expressed, in the same way as for T2, as a multiplicity of elements ( track segments, energy clusters, etc.) specific to each subdetector. For the calorimeters, various thresholds can be applied. The results of the various sub-detectors are transmitted to a Central T3 processor (T3P) which produces the final T3 decision. At the start of a new run, the T3P receives the description of the look-up table of T2. For T3 selection, it simply reproduces in software the T2 algorithm using the results it receives from each LT3P. If it does not confirm the T2 decision the event is simply ecluded from the readout. There is a possibility to enable or disable each trigger component for test purposes. Newly introduced T2 components, not already known to the T3P, are accepted automatically. A small fraction of rejected events and the random T2 trigger component are also accepted for monitoring purposes. The rejection power of T3 depends on the beam conditions and detector noise, and varies between a factor $2 \div 2.5$. The reduction is mainly due to more stringent constraints on the tracking detectors, sharper threshold settings on the calorimeters, as well as noise rejection.

\subsection{The T4 Trigger}

The fourth level trigger of DELPHI was proposed at the beginning of the experiment as a software trigger operating at the last stage of data taking, before the data logging, to either tag rare event candidates or to filter out less interesting events. Its function concentrated on the rejection of those triggers which pass the $\mathrm{T} 1, \mathrm{~T} 2$ hardware and T3 software triggers but contained reduced physics information. The presence of such events is due to several reasons. In particular the geometrical acceptance of the trigger 
subdetectors is bigger than the one in which the reconstruction of tracks or clusters is

performed with sufficient accuracy. This is especially true in the forward regions.

The level-4 trigger was running on 3081/E emulators [7], the first RISC processors developed for high energy physics applications, until 1994. In 1995, with the advent of fast commercial RISC processors, it was implemented on Alpha processors and re-engineered to cope with the LEP2 needs. It was originally running fast simplified detector algorithms but it was since upgraded to process triggers through the complete standard offline reconstruction program (DELANA)[4]. This had the double advantage of guaranteeing that the trigger selection was the same as the one performed offline while simplifying the update and maintenance of the code.

After considering various possible implementations, a scheme was adopted to run T4 in "playback mode", scheduling T4 jobs to process run files ${ }^{5}$ as they are collected through the main data stream. The T4 processes can be executed in any of the fast CPU nodes of the VMS Online Cluster. Up to three T4 processes were running in parallel in 2000, two processes in one dual processor AlphaServer 2100 and one process in one AlphaStation $255 / 233$.

The control of T4 is mainly built on two standard packages, largely used in other online applications:

- An interprocess communication mechanism based on the VMS distributed lock management providing semaphores over the entire Online Cluster;

- The DELPHI Run Data Base (DRDB) and associated library to access the processes.

In practice the $\mathrm{T} 4$ control sequence is the following:

- On completion of a run file, the relevant information is stored in the run database and the T4 scheduler is signalled;

- The scheduler queues the run for subsequent processing;

- At the completion of the processing, the run file is marked as "processed" in the run database and a list of selected triggers is released. The run file is queued for final logging and the data logger is signalled;

- The datalogger logs the selected triggers adding information from the T4 processing to each trigger. On completion, the run file is marked as "logged" and the statistics on the $\mathrm{T} 4$ processing are stored in the run database.

In order to avoid any loss of physics data, the T4 control system includes an exhaustive handling of exceptions as well as a save recovery mechanism of the event in case of a fail in the T4 decision process (e.g. of a hang or loop in the T4 processing, crashes of involved processes or machines, etc.).

The T4 runs the standard offline detector code and performs the standard DELANA tagging. To speed up the processing step, only minor modifications were made to the steering of the reconstruction. The algorithm investigates successively:

- the amount of energy deposition in the electromagnetic calorimeters;

- the results of the pattern recognition in the TPC track detector.

The algorithm stops as soon as a required signature is found in any of these detectors, otherwise it continues with all the other detectors as in the offline. At the end of processing, the triggers are either tagged with the name of the detector for which the algorithm was successful, or are declared empty.

A set of databases is required by the DELANA reconstruction. Some of these, such as

\footnotetext{
${ }^{5} \mathrm{~A}$ run file contains about 3000 triggers gathered in about 15 minutes.
} 
the detector calibration or accelerator parameters databases, are regularly updated during data taking. To take into account these possible changes, the T4 jobs systematically reload these databases before the start of processing of a new run file.

T4 monitoring is performed in two successive steps, one online during data taking and one offline by comparison with the results from an offline processing.

The Data Flow Status is an utility which presents, in real time, the state of the $\mathrm{T} 4$ processes. The status of run files being processed or queued is displayed together with timing information on the processing. Checks are also made to verify the basic T4 parameters e.g. timing and rejection rate. Histograms are produced to allow the study of trends in the behavior of $\mathrm{T} 4$ characteristics such as the tagging rates.

As soon as the offline reconstruction is complete, typically within a day, the results are automatically compared with the ones from the offline. Detailed event-by-event comparison is done to identify any differences between the two processings. Small discrepancies do show up, but at a very low rate. They usually originate from small differences in the databases used by the online and offline processing steps: the databases are sometimes updated a posteriori, after the T4 processing. For monitoring purposes, a small fraction of T4 rejected events is submitted for offline processing and checked to be events without physics interest by the automatic comparison procedure. In 2000, at LEP centre-of-mass energies between 204 and $209 \mathrm{GeV}, 40 \%$ of the triggers submitted to T4 were rejected as empty. This corresponded to a reduction of $27 \%$ in data volume.

An interesting by-product of T4 was the extension of this software to run in a dedicated online farm of six to nine AlphaStation 200/233 for the purposes of checking the quality of data. Instead of running the speeded up version of DELANA as for the T4 trigger, the complete offline reconstruction code is run. The mechanism is identical to the one used for T4, but now only hadronic candidates already, well identified after the T3 step treigger, are processed. During this processing, exhaustive histograms of the reconstructed quantities (e.g. vertex and track parameters, energy releases) are accumulated thus allowing the data quality up to the physical level to be checked in a near real-time. Furthermore, the tagging algorithm which was normally run offline after DELANA to select out candidates for new physics, was also implemented to follow immediately the full DELANA online reconstruction. In case of evidence of interesting candidates, the physicists on shift were alerted. This allowed DELPHI to see the first $W^{+} W^{-}$event ever produced in $e^{+} e^{-}$collisions, within one hour of being taken, in July 1996.

\subsection{The Trigger Data}

The data from the central trigger system are stored in on an event-by-event basis in order to reconstruct, for each event, the subdetectors which caused the trigger. In the pilot record of the event the following information is stored:

- the values of the 120 inputs of the PYTHIA modules for both first and second level;

- the values of the 16 outputs of the PYTHIA modules for both first and second level before and after the prescaling and masking stages;

- the values of the 16 outputs of the T3 processor;

- the values of the prescaling factors acting on the PYTHIA outputs.

With this information it is possible to reconstruct the components and subdetectors which triggered each event. These data are used to estimate the efficiencies, as described in the following. 


\section{Trigger Performance}

\subsection{Efficiency Estimation}

To estimate the efficiency, a sample of events, sensitive to one or more independent triggers beside the one we want to compute the efficiency for, is selected. Defining $N_{T O T}$ as the total number of events and $N_{o b s}$ as the subsample triggered by the component under study, the efficiency $\epsilon$ is estimated as:

$$
\epsilon=\frac{N_{o b s}}{N_{T O T}} .
$$

The mathematical treatment when two or more independent trigger components are present is described in [8].

In the DELPHI trigger configuration for LEP runs at the $\mathrm{Z}$, the logic combinations of subdetectors induce correlations between the trigger components. The way to handle this situation properly was described in [1]. During the high energy runs of LEP a substantial reduction of the trigger components was necessary to moderate the data acquisition rate. The trigger conditions described in section 3.1 show a clear separation between independent parts of the detector. In particular, the forward, barrel and backward regions are now distinct and can be used as independent components in the evaluation of the efficiency. This separation is present both at first and at second level and this by itself takes into account correlations between the different trigger levels. Even though reduced with respect to the Z-running configuration, redundancy is still present in the system and it is exploited to compute the efficiency for event topologies involving only one geometrical region of the detector. It also prevents any data taking inefficiencies arising from potential subdetector malfunctions.

The definition of the efficiency implies the use of the binomial distribution for the estimation of the uncertainty on $\epsilon$. For large samples of events the normal approximation holds and the variance $\sigma^{2}$ can be used to estimate the error $\Delta \epsilon$ :

$$
\sigma^{2}=N_{T O T} \epsilon(1-\epsilon) \text { and } \Delta \epsilon=\frac{\sigma}{N_{T O T}}
$$

Nevertheless this approach is unsatisfactory for small size samples and/or for "very efficient" components for which $\epsilon \rightarrow 1$ and then $\Delta \epsilon \rightarrow 0$. This situation indeed occurs in the data due to either limited statistics or due to the good performance of the trigger system. In such a situation the uncertainty on the efficiency is estimated with the general confidence interval approach which by itself takes into account the characteristics of the binomial distribution and the total sample size. The method used to derive the $68 \%$ confidence limits for the binomial distribution is described in [9]. The complete method is used for samples with $N_{T O T} \leq 50$ and for the cases when $\epsilon \rightarrow 0$ or $\epsilon \rightarrow 1$; otherwise the errors are obtained using the normal approximation.

\subsection{Subdetector and Global Efficiencies}

In the DELPHI system several signals from different subdetectors are combined to form the trigger conditions. From the physics analysis point of view one obviously defines the subdetector efficiency corresponding to a specific topology response. As an example consider the efficiency to single tracks ${ }^{6}$ inside the geometrical acceptance of the subdetector and with special identification requirements from the offline analysis as:

\footnotetext{
${ }^{6}$ See section 4.3 for the definition of this topology and the criteria used to trigger it independently
} 
- muon identification for the muon chambers;

- electron identification for the electromagnetic calorimeter;

- no special requirement for tracking devices.

This, for example, implies analyzing the muon chamber trigger response for tracks identified as muons in the offline analysis. These efficiencies are reported in figures 6 and 7 for the subdetectors in the barrel and forward(backward) regions. The results are shown for tracks inside the geometrical acceptance of each subdetector. A kinematical cut is also applied $\left(P_{T} \geq 3 \mathrm{GeV} / \mathrm{c}\right.$ for tracks and $E \geq 5 \mathrm{GeV}$ for electrons in the calorimeters). The plots report the efficiency as a function of the angular variables $(\theta, \varphi)$. For the backward region the complementary angle $180^{\circ}-\theta$ is used to compare with the forward region. The efficiency is fairly constant and high and follows the geometrical acceptance of the detectors, e.g. for the TPC the reduction corresponding to the transition region between sectors, at $\varphi=30,90,150,210,270$ and 330 degrees, is very clear. A few lower points are known, e.g. for the FEMC in the backward region a lower efficiency is observed; the reason for this is the different electronic noise in the two arms of the calorimeter. To reduce the trigger rate in the FEMC sufficiently more noisy channels were masked in the backward region compared to the forward one.

The subdetector efficiencies cannot be directly translated into efficiencies of the trigger system. To estimate the actual trigger efficiency one has to consider the different logical combinations of trigger signals described in section 3. The results for several event classes of physical interest are described below.

\subsection{Single Track Efficiency}

It is important to estimate the trigger efficiency on isolated tracks as a function of their momentum and direction. These results can be used to obtain the trigger efficiencies for physics channels involving low track multiplicities and low-momentum tracks. This is the case for several $\gamma-\gamma$ analyses and also for some SUSY scenarios (e.g. the degenerate heavy chargino production with small release of visible energy in the apparatus). To estimate these efficiencies an event sample is obtained by selecting isolated tracks which fulfill the following track quality requirements:

- momentum $p \geq 0.2 \mathrm{GeV} / \mathrm{c}$;

- momentum error $\frac{\Delta p}{p} \leq 1$;

- track length $\geq 30 \mathrm{~cm}$;

- impact parameter $r-\varphi \leq 4 \mathrm{~cm}$;

- impact parameter $r-z \leq 10 \mathrm{~cm}$.

The tracks are separated in the following angular regions: (a) forward $\left(10^{\circ} \leq \theta \leq 40^{\circ}\right)$, (b) barrel $\left(40^{\circ} \leq \theta \leq 140^{\circ}\right)$ and (c) backward $\left(140^{\circ} \leq \theta \leq 170^{\circ}\right)$. An independent selection is ensured by requiring a track triggered activity in the regions complementary to the one under study. In figure 8 we report the efficiencies as a function of the transverse momentum of the track. The barrel and forward(backward) regions are different. In the barrel region two different values of the nominal threshold of the TPC-CTG trigger have been used during the LEP2 runs. These values are 0.8 and $1.2 \mathrm{GeV} / \mathrm{c}$, and the results are shown in figure 8(a). The lower threshold was abandoned at the end of the 1998 data taking because the trigger rate was too high $\left(\nu_{T 2} \approx 10 \mathrm{~Hz}\right)$. The results for the forward and backward regions are superimposed and reported in figure $8(\mathrm{~b})$.

To study the angular variation of the efficiency for single tracks, a kinematical cut $P_{T} \geq 3 \mathrm{GeV} / \mathrm{c}$ has been applied. The efficiency, as a function of the $\theta$ angle, is reported 
in figure 9(a). A small reduction, in accord with the results of section 4.2, is observed in the backward region, and the effect of the two TPC halves is visible at $\theta=90^{\circ}$. For the angular variable $\varphi$, the barrel and forward(backward) regions have been separated. $\mathrm{h}$ e results for the barrel are shown in figure 9(b). We show the results for the complete trigger and after having removed the $\mathrm{IDOD}_{6}$ component which matches the TPC cracks. A clear recovery is observed apart from some small regions where known OD inefficiencies are present. The results for the forward and backward regions are superimposed in figure $9(\mathrm{c})$.

\subsection{Single Photon Efficiency}

The trigger efficiency for single photons can be derived analogously to the single track efficiencies. We select events having an isolated track identified as an electron in the following angular regions: (a) forward $10^{\circ} \leq \theta \leq 35^{\circ}$, (b) barrel $45^{\circ} \leq \theta \leq 135^{\circ}$, (c) backward $145^{\circ} \leq \theta \leq 170^{\circ}$. As usual, the event is required to have satisfied another independent trigger from the one in question.

For the barrel region the efficiency as a function of the electron energy is shown in figure 10(a). The plateau, $\epsilon \sim 80 \%$ at high energy, is limited by several factors. The trigger is formed with the coincidence of the scintillator response inside each HPC module and the response from the chamber data. The geometrical acceptance is reduced by the cracks due to the $\varphi$ and $\theta$ segmentations (24 and 6 sectors respectively). Moreover the geometrical acceptance inside each module is further reduced by the dimension of the scintillator which does not match exactly the dimension of the modules. This effect accounts for $\sim 5 \%$ of inefficiency. The other inefficiency is due to very low efficiency in a few modules. This is shown in figure 10(b) in which the efficiency as function of the $\varphi$ angle is reported for electrons with $E \geq 10 \mathrm{GeV}$. The segmentation is choosen to match the one of the detector modules. Separate results are shown for the $\theta \geq 90^{\circ}$ and $\theta \leq 90^{\circ}$ regions. Due to the trigger logic each $\varphi$-sector shown in the figure corresponds to three HPC modules along $\theta$. The efficiency in each $\varphi$-sector reflects the performance of the three modules. There are two reasons for the bad modules, namely problems with scintillator light transmission with optical fibers and problems of noise in several modules which had to be switched off both for scintillators and chamber data. The overall inefficiency is due to important losses in a few modules.

For the forward and backward regions the results are shown in figure 11. The threshold in the FEMC calorimeter was changed from 3 to $5 \mathrm{GeV}$ after the 1998 data taking to moderate the rate. The efficiency as a function of the energy is shown in figure 11(a-b) for the forward and backward regions respectively. The angular distribution of the efficiency is studied having applied a kinematical cut $E \geq 10 \mathrm{GeV}$. The results are shown in figure $11(\mathrm{c})$ for the $\varphi$ angle and in figure $11(\mathrm{~d})$ for the $\theta$ angle. Due to the noise problem mentioned above, a difference in efficiency is observed between the forward and backward regions, at high energies $E \geq 10 \mathrm{GeV}$ the value is $\Delta \epsilon=0.020 \pm 0.002$.

\subsection{Efficiency for Dilepton Events: $e^{+} e^{-} \rightarrow e^{+} e^{-}, \mu^{+} \mu^{-}, \tau^{+} \tau^{-}$}

The dilepton samples consist of $e^{+} e^{-}, \mu^{+} \mu^{-}$and $\tau^{+} \tau^{-}$events identified requiring standard DELPHI cuts [4]. In these events the topology is characterized by two, generally in a back-to-back configuration, localized clusters: high momentum tracks and/or electromagnetic energy. Depending on to the geometrical distribution of the two clusters, it is possible to define in each case two independent components to estimate the trigger 
efficiency. This is shown schematically in figure 12 . The angular trigger efficiency results are shown in figure 13(a-c). For $e^{+} e^{-}$and $\mu^{+} \mu^{-}$events the polar angle used is the average $\theta=\left(\theta^{+}+\pi-\theta^{-}\right) / 2$. For the $\tau^{+} \tau^{-}$pairs the results are shown as a function of the polar angle of the thrust axis.

\subsection{Efficiency for Hadronic Events}

For the hadronic events the selection is described in [4]. For this topology the event polar angle is taken to be that of the thrust axis. Similarly to the case of the leptonic events two independent trigger components are defined in the different $\theta$-regions in order to determine the efficiency. The results are reported in figure 13(d).

\subsection{Efficiency for Multiphoton Events}

The multiphoton events $e^{+} e^{-} \rightarrow \gamma \gamma(\gamma \ldots)$ provide an important test for new physics phenomena [10]. As reported in section 3.1, the trigger for these events utilizes the single photon components, the dedicated component of which uses only the scintillator data in the HPC and the TOFBB. The efficiency of the different components for these events can be studied in Bhabha events at large angles. The results are shown in figure 13(e).

\section{Conclusions}

We have presented the modifications that were introduced in the DELPHI trigger complex since the beginning of the high energy runs of LEP. The configuration of the trigger conditions has been discussed and the performances have been shown. Very good efficiency and acceptance rates have been obtained.

\section{Acknowledgements}

We would like to thank all our DELPHI colleagues involved in the data taking and in the detector activities for their continuous help during the running periods. 


\section{${ }_{404}$ References}

405 [1] V.Bocci et al., Nucl. Instr. and Meth. A362 (1995) 361

${ }_{406}^{2}$ [2] DELPHI Collaboration: P. Abreu et al., Phys. Lett. B515 (2001) 238

${ }_{407}$ [3] DELPHI Collaboration: P. Abreu et al., Phys. Lett. B485 (2000) 95

${ }_{408}$ [4] DELPHI Collaboration: P. Abreu et al., Nucl. Instr. and Meth. A378 (1996) 57

409 [5] S. Quinton et al., IEEE Trans. Nucl. Sci. 36 (1989) 390

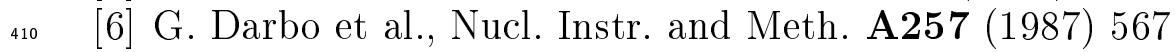

${ }_{411}^{2}$ [7] The 3081/E Emulator, CCC/CERN 84-9 and CERN/DD 85-16

[8] A.Frodesen et al., Probability and Statistics in Particle Physics, Universitetforlaget, Bergen (Norway), 1979, p.20

[9] W.T. Eadie et al., Statistical Methods in Experimental Physics, North-Holland Publishing Company, 1971, p.200

${ }_{414}^{410]}$ DELPHI Collaboration: P. Abreu et al., Eur. Phys. J. C17 (2000) 53 


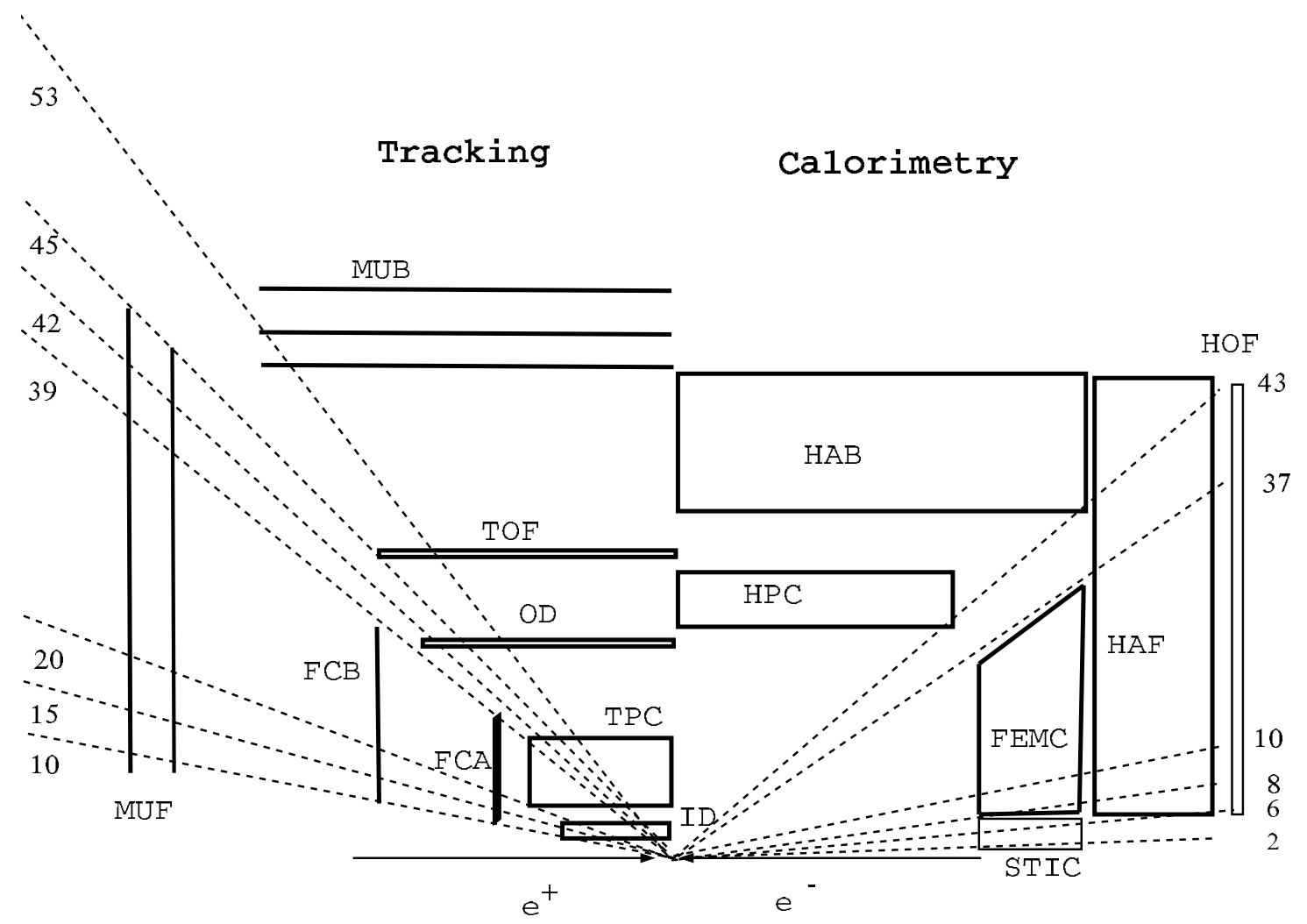

Figure 1: The subdetectors participating in the trigger decision and their acceptance in polar angle $\theta$ (in degrees). The system involves tracking devices (ID, OD, TPC, FCA and FCB), electromagnetic calorimeters (HPC, FEMC and STIC), hadron calorimeters (HAB and HAF), scintillators (TOF and HOF) and muon chambers (MUB and MUF). To simplify the figure only half of the subdetectors are shown. 


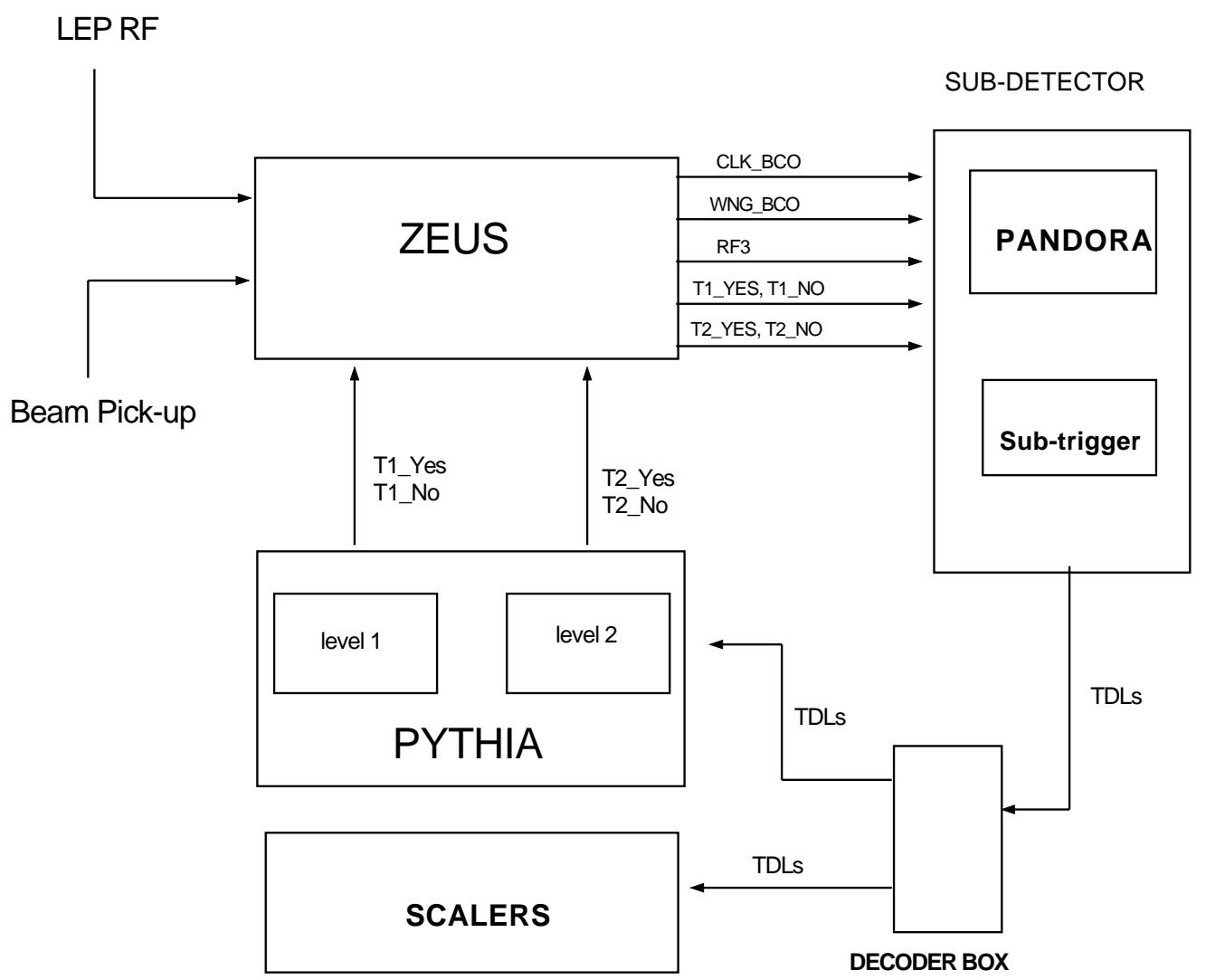

Figure 2: Schematic view of the hardware architecture of the DELPHI trigger system. 


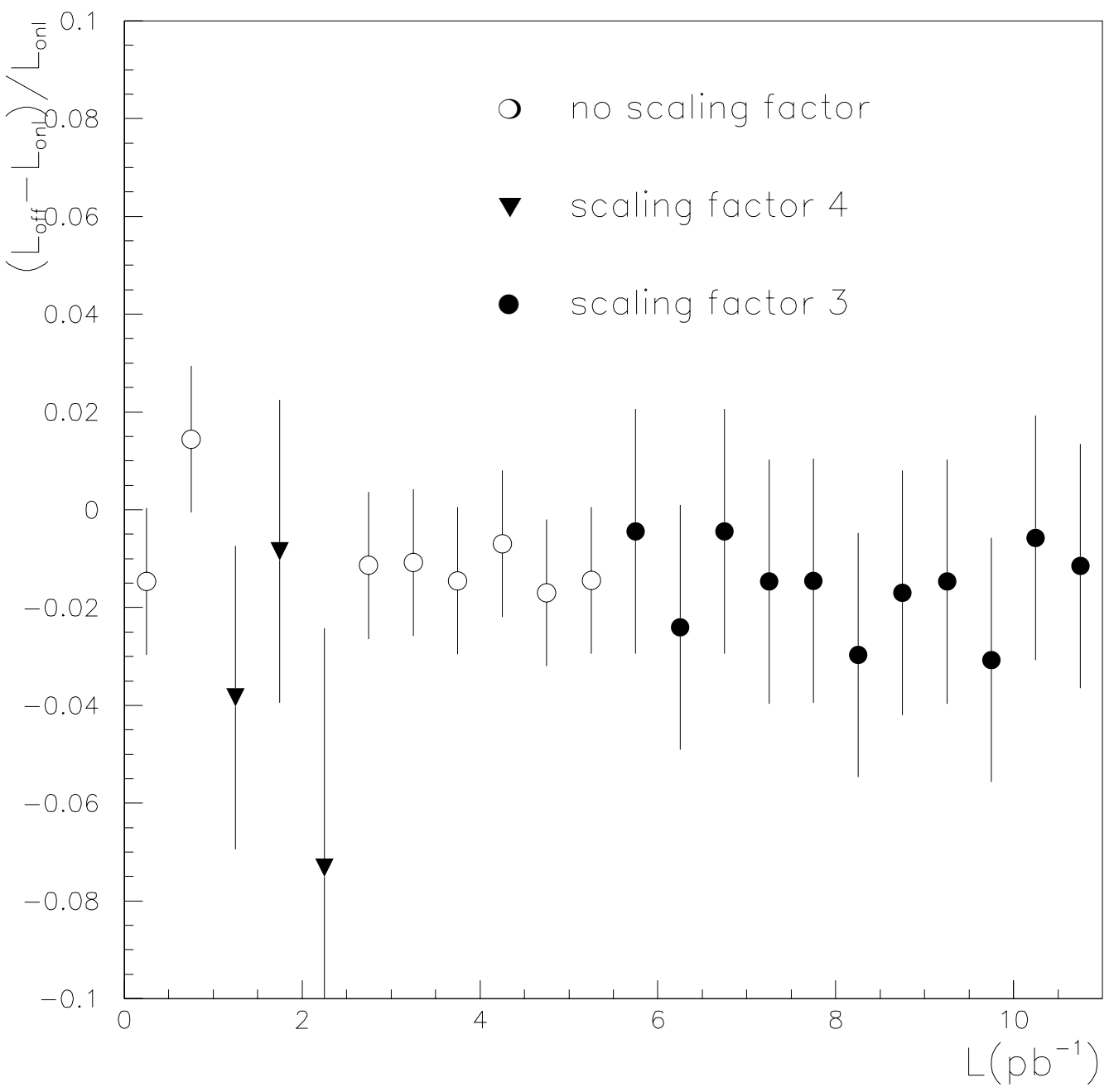

Figure 3: The fractional difference between the integrated luminosity obtained offline from Bhabha events, after the correction for the prescaling factors, and the online luminosity obtained from the scalers as a function of the total integrated luminosity. These results are for the 1996 data at $\sqrt{s} \approx 160 \mathrm{GeV}$. A stable systematic difference of $\sim 1.5 \%$ is present because the online measurement does not include the full set of used offline. 


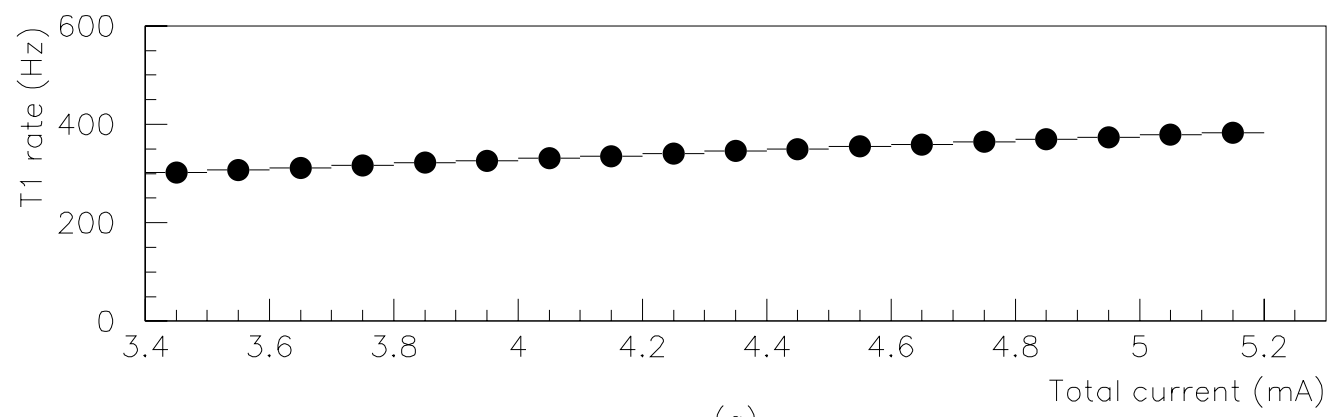

(a)

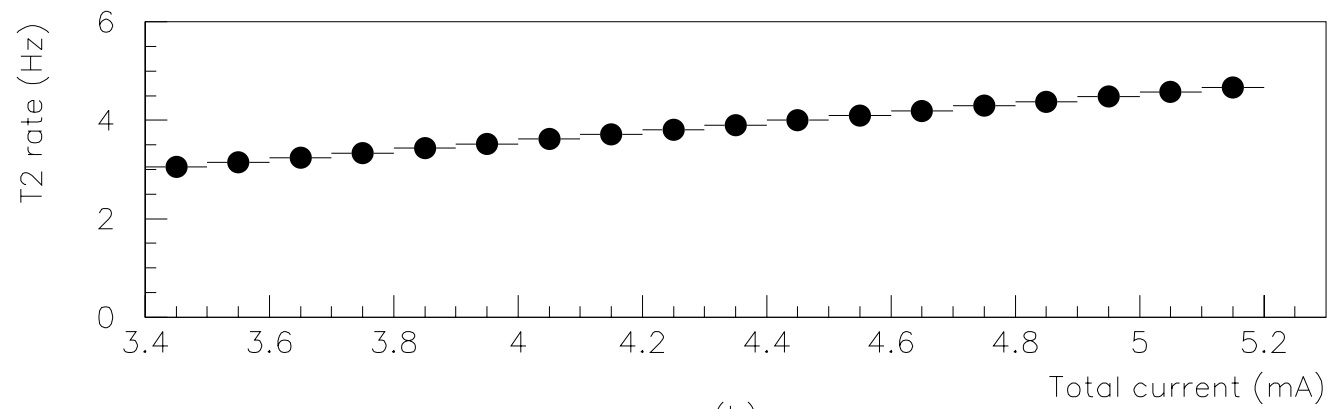

(b)

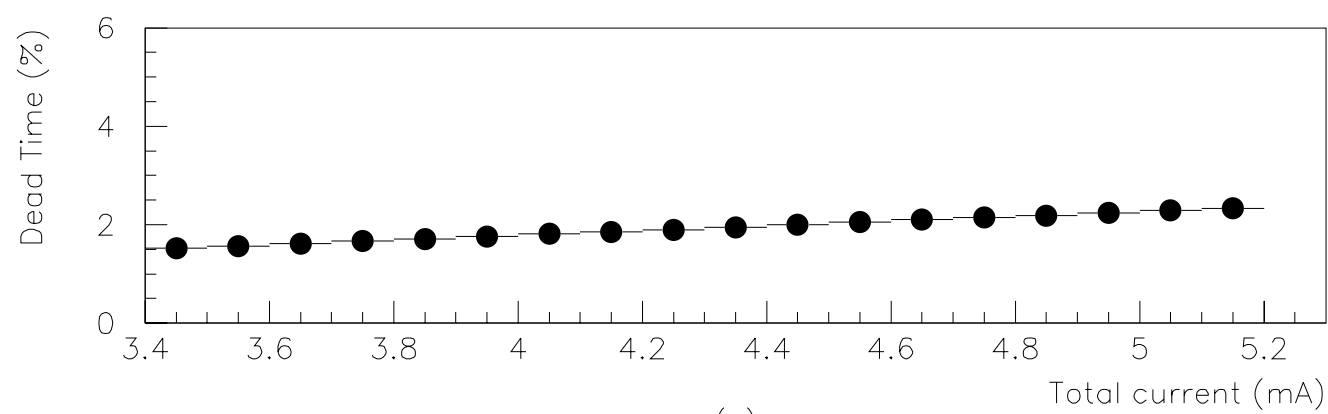

(c)

Figure 4: (a) T1 trigger rate as a function of the total LEP current, (b) T2 trigger rate as a function of the total LEP current, (c) Fractional dead-time induced by the trigger as a function of the total LEP current. The data correspond to the 2000 data-taking period. 

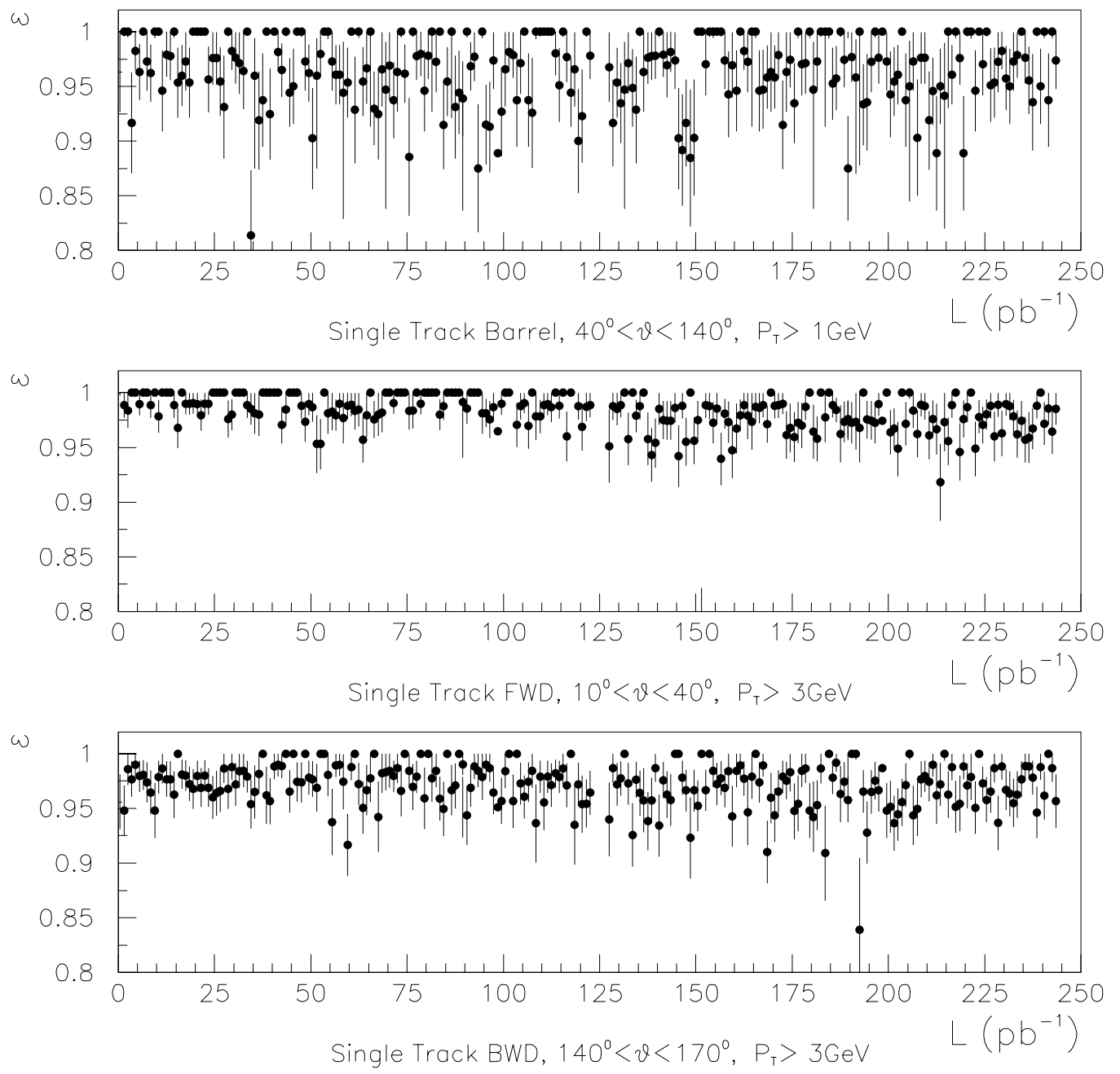

Figure 5: Stability of the trigger system. For single-track triggers in the different angular regions, the efficiency is shown as a function of the total collected integrated luminosity. Data from the 1999 period are reported. The bin width is $\sim 1 \mathrm{pb}^{-1}$ in order to have sufficient statistics and corresponds to $\sim 1$ day of data taking with stable machine conditions. The low points correspond to malfunctioning of some subdetectors. 
Barrel Region
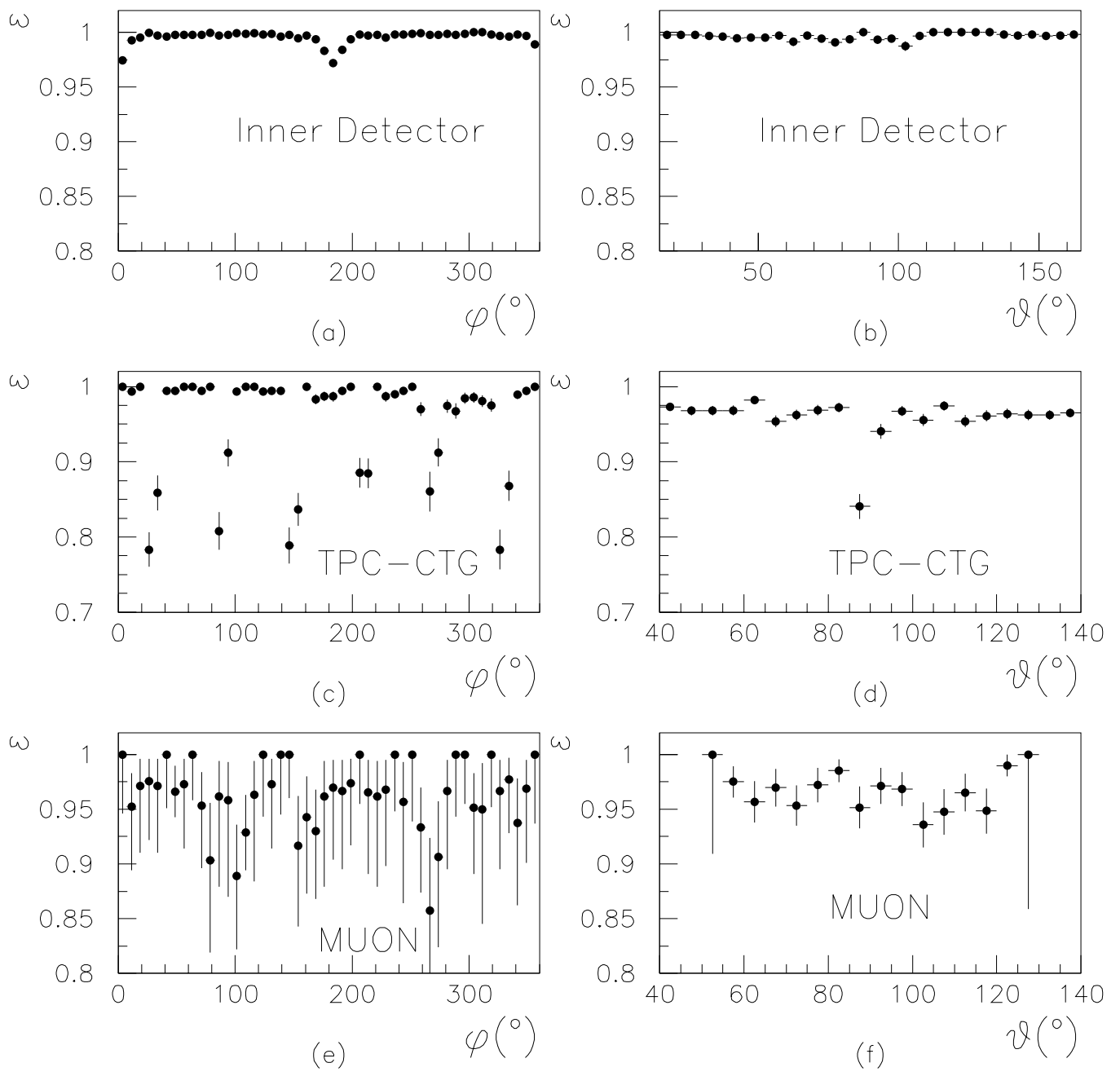

Figure 6: Subdetector trigger efficiency in the barrel region for single tracks with $P_{T} \geq 3 \mathrm{GeV} / \mathrm{c}$ 

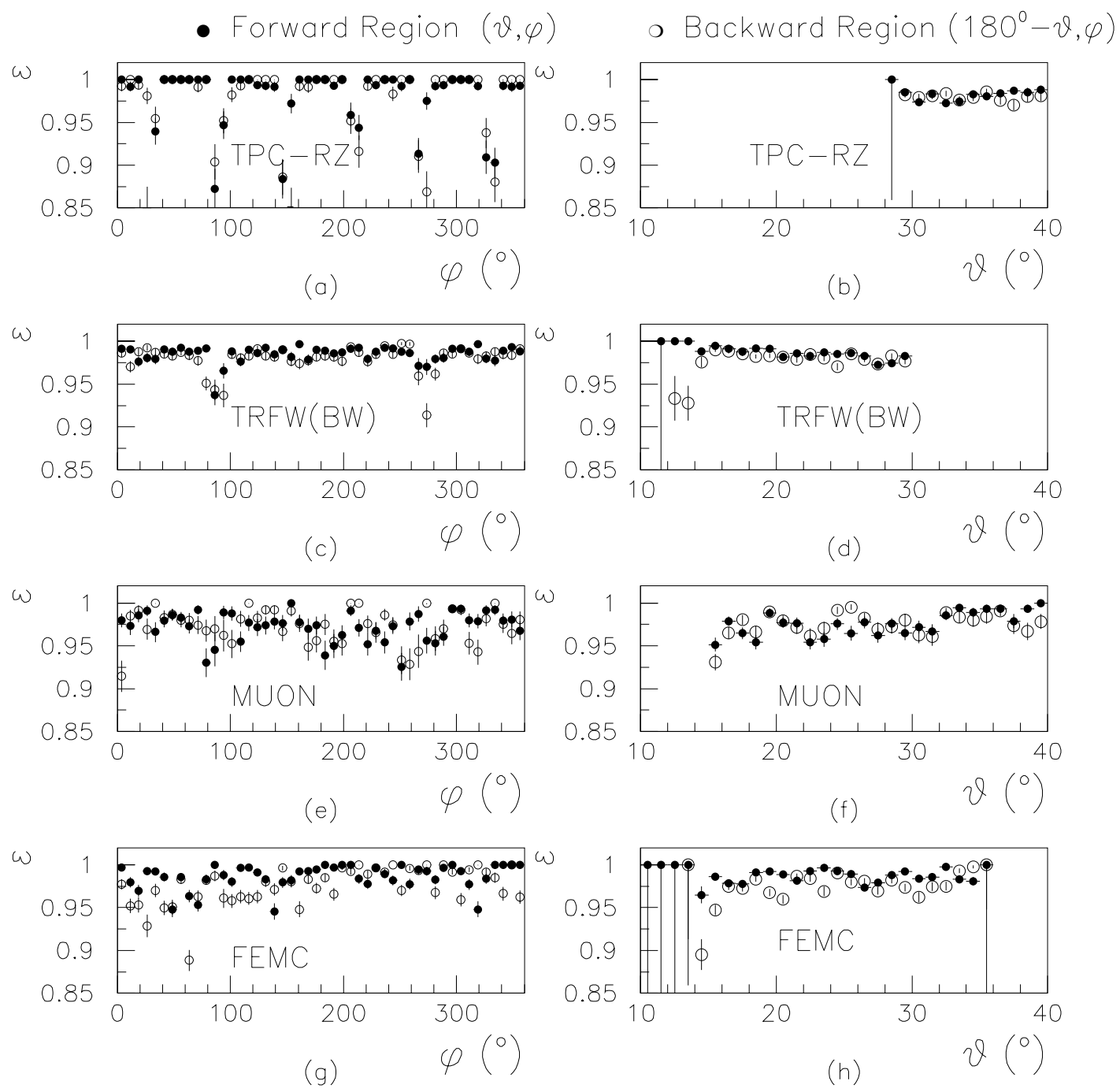

Figure 7: Subdetector trigger efficiency in the forward and backward regions for single tracks with $P_{T} \geq 3 \mathrm{GeV} / \mathrm{c}(P \geq 5 \mathrm{GeV} / \mathrm{c}$ for FEMC). Data from forward and backward subdetectors are superimposed. In the backward region the complementary $\left(180^{\circ}-\theta\right)$ angle is reported to allow the comparison. 

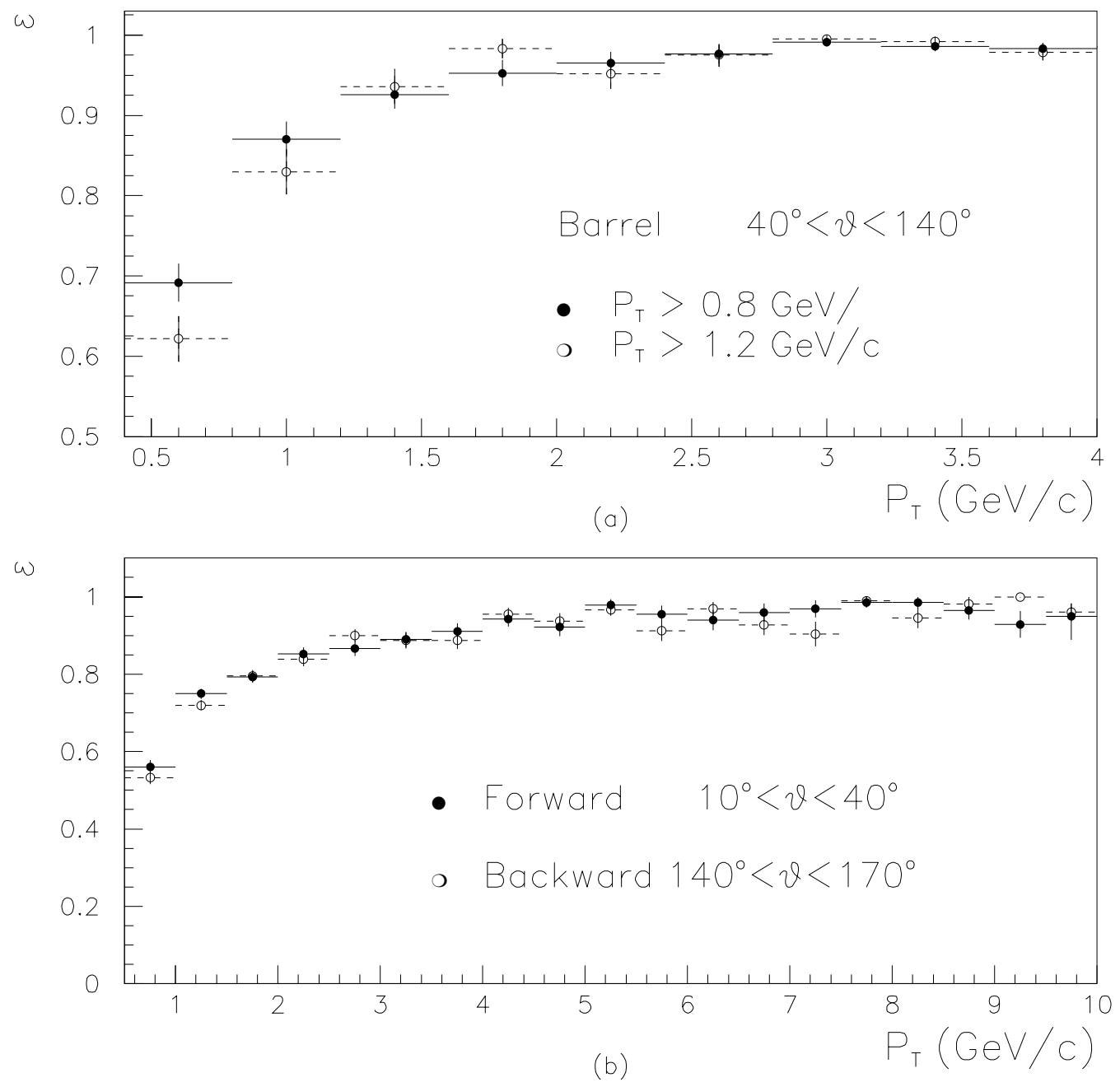

Figure 8: Trigger efficiencies for single tracks as a function of the transverse momentum: (a) in the barrel region, two values of the TPC-CTG threshold have been used during the high energy runs and the results are reported; (b) the forward and backward regions results are superimposed. 

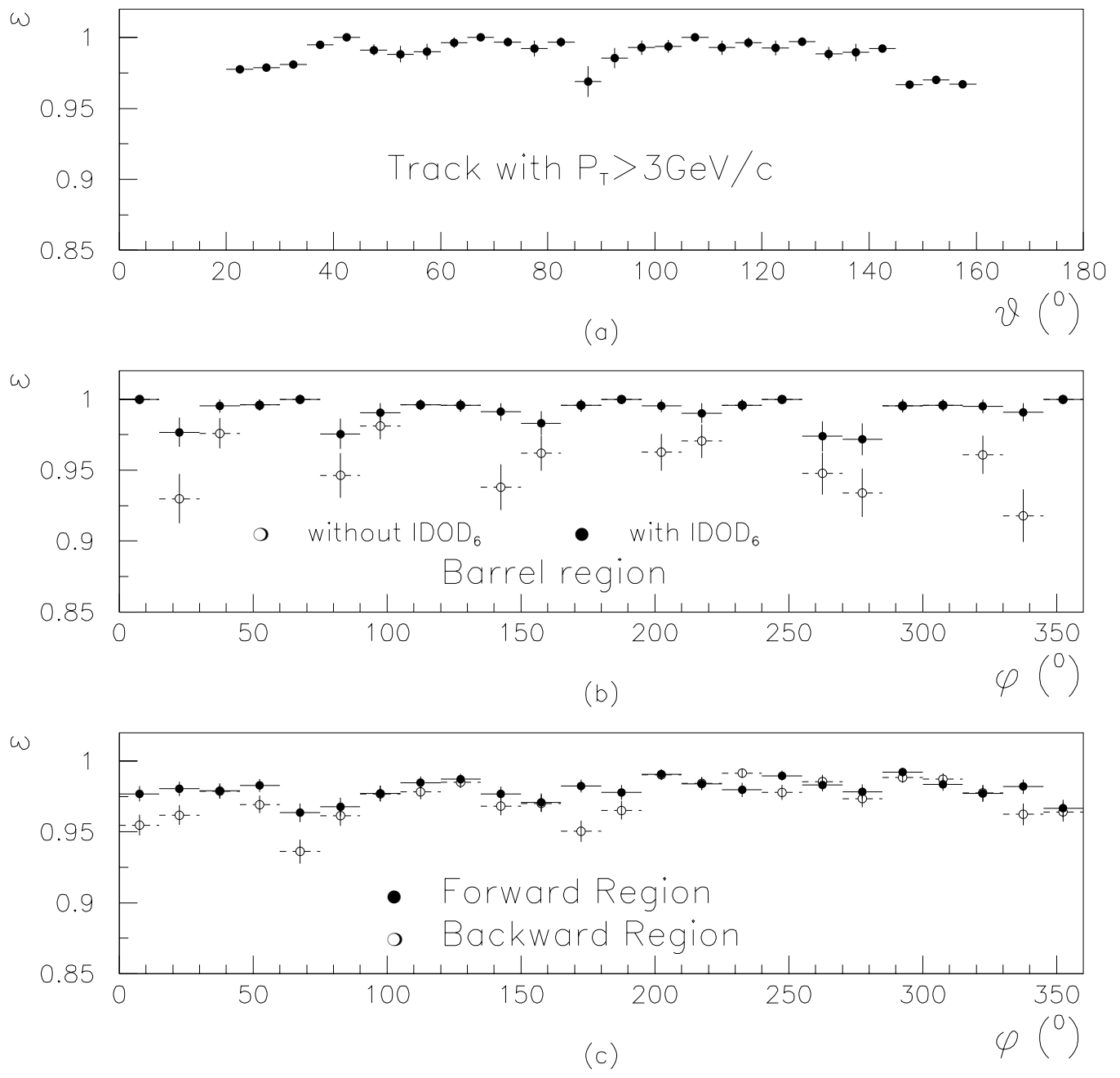

Figure 9: Trigger efficiencies for single tracks as a function of the angular distribution. A transverse momentum cut of $3 \mathrm{GeV} / \mathrm{c}$ is applied: (a) $\epsilon$ versus $\theta$, (b) $\epsilon$ versus $\varphi$ in the barrel region, the results for the total trigger and the trigger excluding the $\mathrm{IDOD}_{6}$ coincidence to cover the TPC cracks are shown and a clear recovery is observed; (c) $\epsilon$ versus $\varphi$ in the forward and backward regions. 

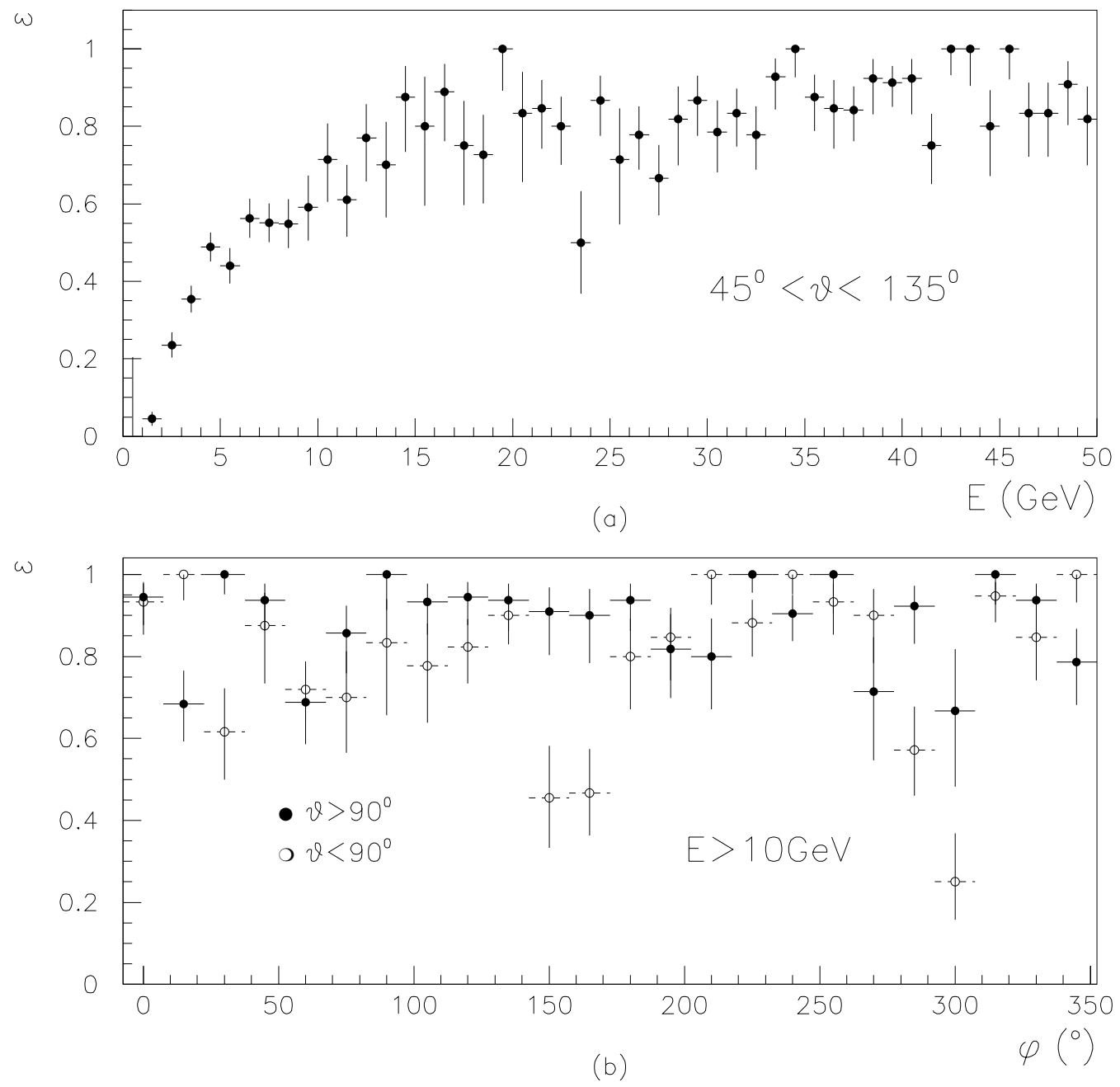

Figure 10: Trigger efficiencies for single photons in the barrel. 

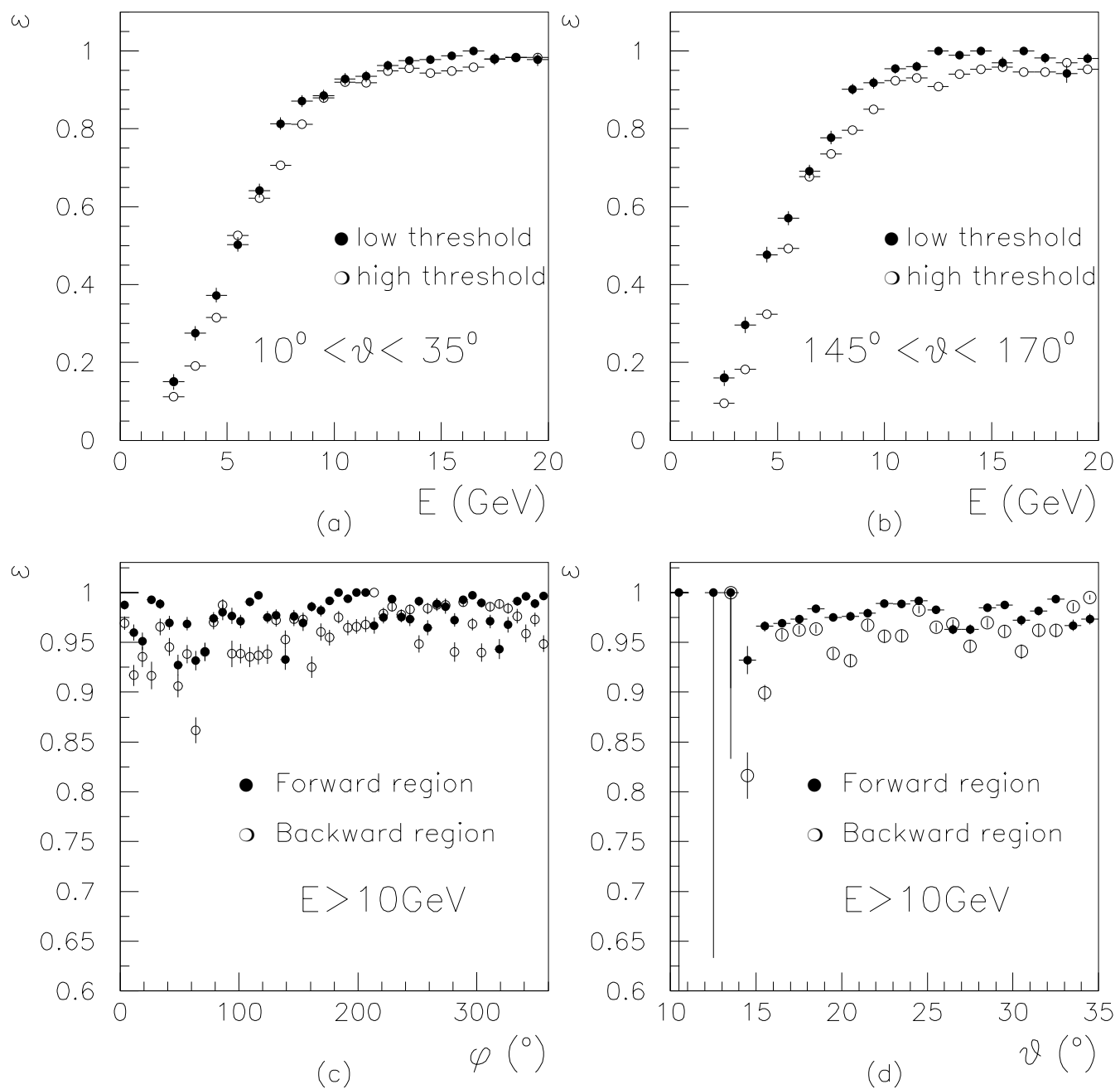

Figure 11: Trigger efficiencies for single photons as derived from electrons in the forward and backward regions. In (a) and (b) the efficiency, $\epsilon$, is plotted as a function of the electron energy $E$ which is derived from the momentum of the track. Two different nominal thresholds have been used before and after 1999 data taking. The corresponding results are superimposed. For the angular distributions a kinematical cut at $10 \mathrm{GeV}$ has been applied: (c) $\epsilon$ versus $\varphi$ in the forward and backward regions are superimposed; (d) $\epsilon$ versus $\theta$ data from forward and backward regions are superimposed using the complementary angle $\left(180^{\circ}-\theta\right)$ for the backward one. As mentionned in the text a difference $\Delta \epsilon=0.020 \pm 0.002$ is observed between the forward and backward regions. 


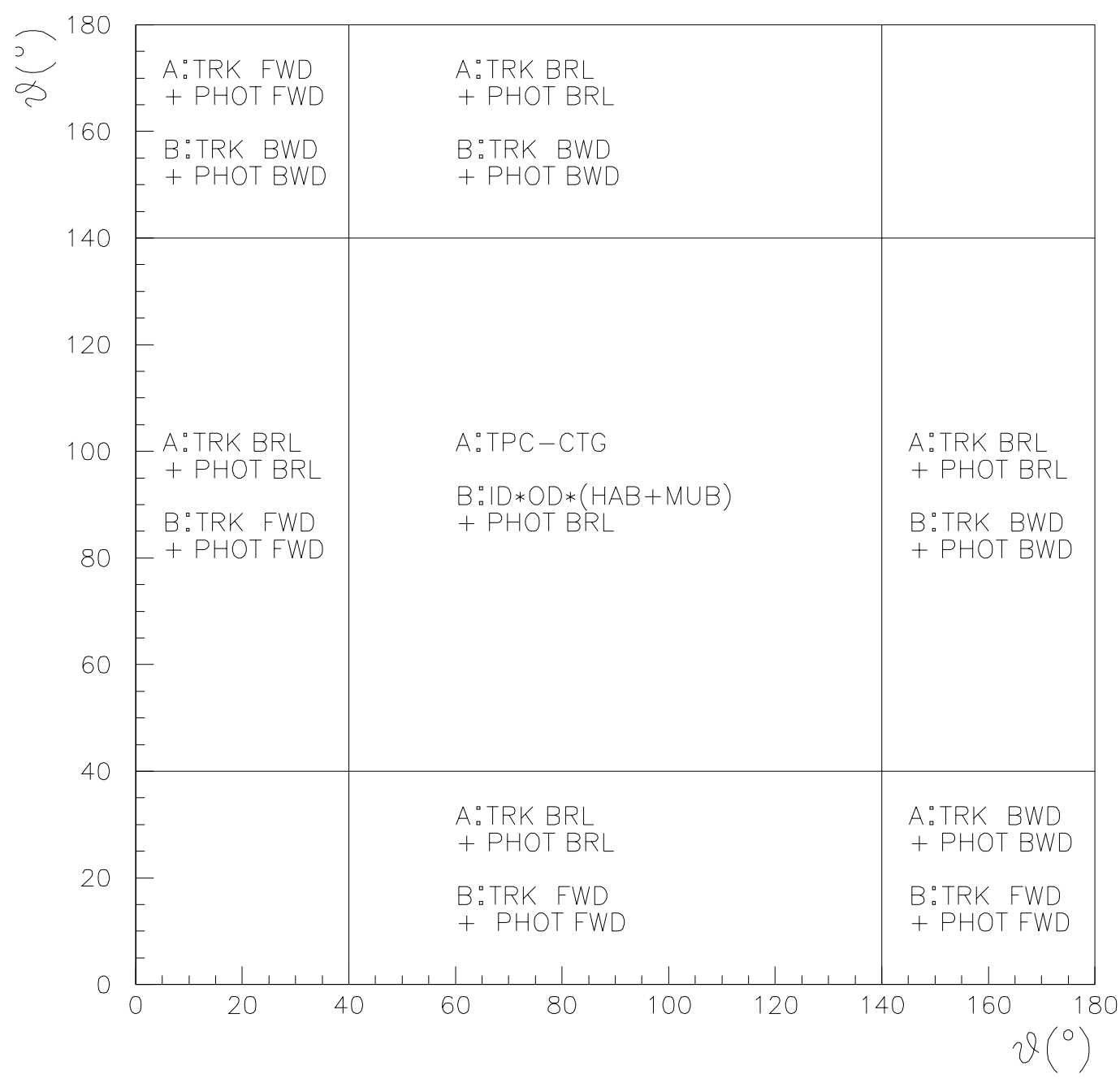

Figure 12: Independent trigger components for dilepton events for the different geometrical topologies. The following abbreviations are used: TRK for single track trigger, PHOT for single photon trigger (see section 3), BRL for barrel, FWD(BWD) for forward (backward) and the names of subdetectors correspond to the ones in figure 1. 


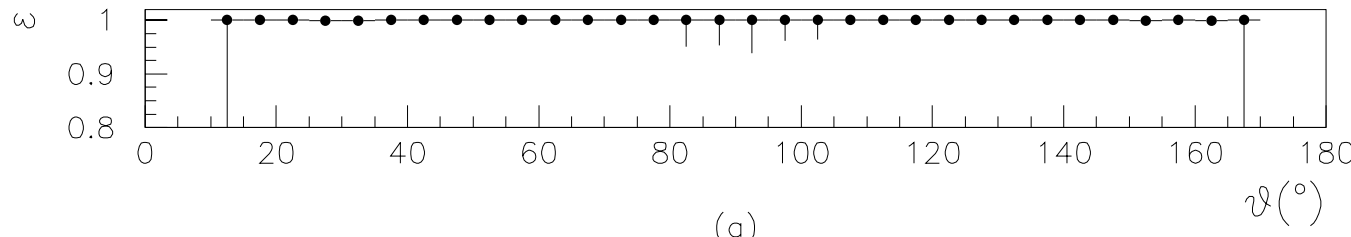

(a)

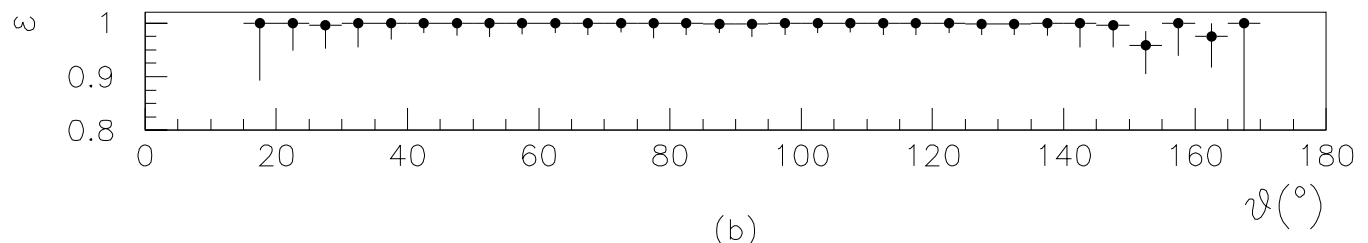

(b)
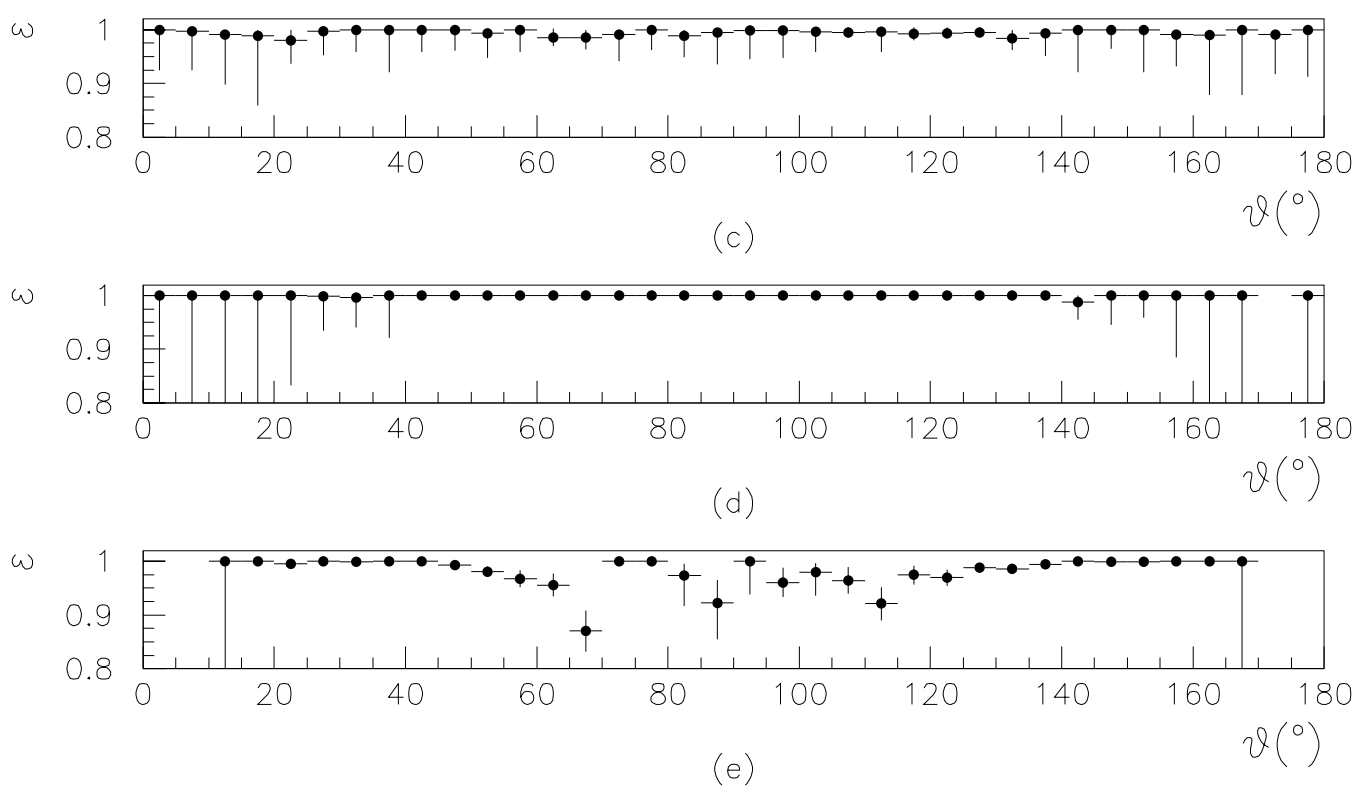

Figure 13: Angular dependence of the trigger efficiency for different classes of events: (a) $e^{+} e^{-}$, (b) $\mu^{+} \mu^{-}$, (c) $\tau^{+} \tau^{-}$, (d) Hadronic events and (e) $\gamma \gamma(\gamma \ldots$. . In the plots (a), (b) and (e) the angle $\theta=\left(\theta^{+}+\pi-\theta^{-}\right) / 2$ is used, while in (c) and (d) the $\theta$ of the thrust axis is used. 